\title{
Perfil clínico-epidemiológico del síndrome metabólico en adultos atendidos en el hospital I Florencia de Mora EsSALUD
}

Yeny Ofelia Tejada López* 1; Gloria María Choquehuanca Zambrano ${ }^{3}$; Evelyn del Socorro Goicochea Ríos ${ }^{1}$; Jessica Ericka Vicuña Villacorta ${ }^{1}$; Olga Yanet Guzmán Aybar ${ }^{1,2}$

\section{RESUMEN}

Objetivo: Determinar el perfil clínico-epidemiológico del síndrome metabólico en adultos atendidos en el Hospital I Florencia de Mora EsSALUD.

Materiales y métodos: Estudio de tipo descriptivo, observacional y retrospectivo en una población de 4752 pacientes tamizados en el programa Reforma de Vida. Se aplicaron los criterios diagnósticos del síndrome metabólico de la Federación Internacional de Diabetes.

Resultados: La prevalencia del síndrome metabólico es de 38,97\%. En el perfil clínico, las variables más frecuentes fueron la obesidad central $(97,50 \%)$, un valor bajo de colesterol HDL $(86,40 \%)$ y la hipertrigliceridemia $(80,10 \%)$. Todas ellas mostraron significación estadística. En el perfil epidemiológico, las variables sexo femenino (68,10\%) y la edad "adulto maduro" (48,30\%) fueron las más frecuentes.

Conclusiones: Más de la tercera parte del total de la población estudiada presenta síndrome metabólico. En este grupo predominan la obesidad, el colesterol HDL bajo, la hipertrigliceridemia, la edad del adulto maduro y el sexo femenino.

Palabras clave: Adulto; Síndrome metabólico; Perfil de salud (Fuente: DeCS BIREME).

\section{Clinical and epidemiological profile of the metabolic syndrome among adults treated at the Hospital I Florencia de Mora EsSalud}

\section{ABSTRACT}

Objective: To determine the clinical and epidemiological profile of the metabolic syndrome among adults treated at the Hospital I Florencia de Mora EsSalud.

Materials and methods: A retrospective, observational and descriptive study conducted in a population of 4,752 patients screened for the Reforma de Vida program. The International Diabetes Federation's diagnostic criteria for metabolic syndrome were used.

Results: The prevalence of metabolic syndrome is $38.97 \%$. The most frequent variables of the clinical profile were central obesity (97.50 \%), low levels of HDL cholesterol (86.4\%) and hypertriglyceridemia (80.10\%). All these variables showed statistical significance. The most frequent variables of the epidemiological profile were female sex $(68.10 \%)$ and middleage adult (48.30\%).

Conclusions: More than one third of the study population has metabolic syndrome. Obesity, low levels of HDL cholesterol, hypertriglyceridemia, middle-age adult and female sex prevailed in this group.

Keywords: Adult; Metabolic syndrome; Health profile (Source: MeSH NLM).

1 Universidad César Vallejo. Trujillo, Perú.

2 Hospital I Florencia de Mora-EsSALUD. Trujillo, Perú.

3 Universidad Privada Anteno Orrego. Trujillo, Perú.

*Autor corresponsal. 
Yeny Ofelia Tejada López; Gloria María Choquehuanca Zambrano; Evelyn del Socorro Goicochea Ríos; Jessica Ericka Vicuña Villacorta; Olga Yanet Guzmán Aybar

\section{INTRODUCCIÓN}

El síndrome metabólico (SM) es una enfermedad multifactorial caracterizada por componentes genéticos que predisponen a almacenar tejido graso (1) y factores adquiridos, como los estilos de vida, que potencian las alteraciones fisiológicas y conllevan a desarrollar diabetes, enfermedades cardiovasculares ${ }^{(2,3)}$ y cáncer ${ }^{(3)}$.

Entre los factores de riesgo para la aparición del SM están la obesidad ${ }^{(2,4-6)}$, la hipertrigliceridemia ${ }^{(4,5,7)}$, el colesterol HDL bajo (HDL, por sus siglas en inglés, high-density lipoprotein ${ }^{(8)}$, la hiperglicemia, la presión arterial alta ${ }^{(4,7)}$, la resistencia a la insulina ${ }^{(4)}$, el aumento del perímetro de la circunferencia abdominal ${ }^{(7,9)}$, el índice de masa corporal mayor o igual a $25^{(10-13)}$, el menor nivel educativo (11,12), el consumo de tabaco ${ }^{(12)}$, el aumento de la edad ${ }^{(10,13,14)}$, el sedentarismo, un antecedente de SM familiar, la diabetes gestacional, el síndrome de ovario poliquístico, las enfermedades mentales y el consumo de medicamentos antipsicóticos ${ }^{(9)}$.

Los criterios diagnósticos del síndrome metabólico han evolucionado con el tiempo. En 1988, el Dr. Gerald Reaven lo describe como el síndrome $X^{(2,15)}$, cuyos componentes eran la resistencia insulina, la hiperinsulinemia, la disminución de colesterol HDL, y el aumento de triglicéridos, colesterol LDL (de sus siglas en inglés low-density lipoprotein) y presión arterial. En 1998, la Organización Mundial de la Salud (OMS) lo denominó como síndrome metabólico (SM), que fue la primera definición unificada ${ }^{(15)}$, y agregó los criterios de índice de masa corporal y microalbuminuria, y excluyó el colesterol LDL (2).

Los diferentes consensos internacionales ${ }^{(8)}$ unificaron los criterios como la alteración de la distribución de grasa corporal, la hipertensión arterial, la hipertriglicéridemia, el colesterol HDL bajo, la hiperglicemia, la resistencia a la insulina, la tolerancia a la glucosa y la microalbuminuria (4). Los consensos NCEP ATP III (de las siglas en inglés, National Cholesterol Education Program y Adult Treatment Panel III) y de la Federación Internacional de Diabetes (IDF, del inglés International Diabetes Federation) han sido los más utilizados ${ }^{(16)}$ por ser más accesibles ya que no incluyen dentro de sus criterios la prueba de resistencia a la insulina, tolerancia a la glucosa y microalbuminuria ${ }^{(14,17)}$.Sin embargo, el NCEP ATP III no toma en cuenta la diferencia del perímetro abdominal que se observa entre los grupos étnicos que sí considera la IDF, ya que uno de sus criterios diagnósticos es el perímetro abdominal de la población cuerdo a la población tamizada, haciendo énfasis el corte en la población latinoamericana ${ }^{(18,19)}$.
En los últimos años el sedentarismo y el consumo de alimentos rápidos hipercalóricos han cambiado la vida saludable de millones de personas ${ }^{(15)}$, y han aumentado la prevalencia de SM en la población adulta, de un $6,6 \%$ hasta el $63 \%(5,11-13,20)$; mientras que en el Perú fluctúa entre el $10 \%$ a un $45 \%$. Además, el SM multiplica por cinco el riesgo de padecer diabetes mellitus tipo 2, duplica el desarrollo de la enfermedad cerebral vascular, y hasta triplica o cuadriplica el riesgo de sufrir un infarto al miocardio ${ }^{(18)}$; con lo que alcanza una mortalidad del $80 \%$ por complicaciones cardiovasculares ${ }^{(2)}$. Por lo tanto, el SM es considerado como la epidemia de este siglo ${ }^{(7,11)}$.

\section{MATERIALES Y MÉTODOS}

\section{Diseño y población de estudio}

Estudio de tipo descriptivo, observacional y retrospectivo. Se incluyó una población de $\mathbf{4 7 5 2}$ pacientes, tamizados en la consulta del programa Reforma de Vida del Hospital I Florencia de Mora (HIFM) EsSALUD (La Libertad) durante el periodo de enero 2014 a diciembre 2017.

\section{Variables y mediciones}

Para valorar el perfil clínico epidemiológico se empleó una ficha para registrar los criterios diagnósticos para SM de la IDF: presión arterial elevada, obesidad central, colesterol HDL bajo, hipertrigliceridemia e hiperglicemia; así como las variables sexo y edad.

\section{Análisis estadístico}

Se utilizó la estadística descriptiva para el análisis de tablas de frecuencias de una entrada y doble entrada, así como las pruebas estadísticas de ji al cuadrado y Odds ratio para las variables del perfil clínico.

\section{Consideraciones éticas}

Se respetaron las normas éticas en medicina, la recolección y análisis de datos. El estudio contó con la autorización del servicio asistencial, fue de carácter confidencial y anónimo, y solo el personal investigador tuvo acceso a la información.

\section{RESULTADOS}

El estudio incluyó una población de 4752 adultos inscritos y evaluados en el Programa Reforma de Vida durante el periodo de estudio. Se excluyeron 56 historias clínicas incompletas, por lo cual los resultados corresponden a 4696 personas. La prevalencia de SM en adultos atendidos en el HIFM - EsSALUD de Trujillo fue de 38,97 \% y se muestra tabla 1. 
Perfil clínico-epidemiológico del síndrome metabólico en adultos atendidos en el hospital I Florencia de Mora EsSALUD

Tabla 1. Prevalencia del síndrome metabólico en adultos. HIFM EsSALUD -Trujillo, 2014 -2017

\begin{tabular}{ccccccc}
$\begin{array}{c}\text { Diagnóstico de } \\
\text { síndrome } \\
\text { metabólico }\end{array}$ & Ausente & \multicolumn{2}{c}{ Presente } & \multicolumn{2}{c}{ Total } \\
\hline & $\mathrm{N}^{\circ}$ & $\%$ & $\mathrm{~N}^{\circ}$ & $\%$ & $\mathrm{~N}^{\circ}$ & $\%$ \\
\hline Total & 2866 & 61,03 & 1830 & 38,97 & 4696 & 100,00 \\
\end{tabular}

En la tabla 2 se encuentran las variables del perfil clínico de SM. Se observa que todas estas variables tienen una asociación estadísticamente significativa con el SM (valor $p=0,00$ ), las más frecuentes son la obesidad central presente en $97,50 \%$, HDL colesterol bajo en $86,40 \%$ y la hipertigliceridemia en $80,10 \%$ de participantes. Además, estas variables son factores de riesgo de acuerdo al OR: obesidad central $(11,88)$; colesterol HDL $(6,28)$; e hipertigliceridemia $(18,6)$. Con las variables presión arterial y glicemia se hizo el diagnóstico de hipertensión arterial $(13,30 \%)$ y diabetes mellitus al $(5,00 \%)$.

Tabla 2. Perfil clínico del síndrome metabólico en adultos. HIFM EsSALUD -Trujillo, 2014-2017

\begin{tabular}{|c|c|c|c|c|c|c|c|c|c|}
\hline \multirow[t]{3}{*}{ Variables } & & \multicolumn{4}{|c|}{$\begin{array}{l}\text { Diagnóstico de } \\
\text { síndrome metabólico }\end{array}$} & \multicolumn{2}{|c|}{ Total } & \multirow[t]{2}{*}{$\mathrm{X}^{2}$} & \multirow[t]{2}{*}{ OR } \\
\hline & & \multicolumn{2}{|c|}{ No $(2866)$} & \multicolumn{2}{|c|}{ Sí (1830) } & \multirow[b]{2}{*}{$\mathrm{N}^{\circ}$} & \multirow[b]{2}{*}{$\%$} & & \\
\hline & & $\mathrm{N}^{\circ}$ & $\%$ & $\mathrm{~N}^{\circ}$ & $\%$ & & & $\mathrm{~N}^{\circ}$ & $\%$ \\
\hline \multirow{4}{*}{ Presión arterial } & HTA & 79 & 2,80 & 243 & 13,30 & 322 & 6,90 & \multirow{4}{*}{$\begin{array}{l}564,75 \\
p=0,00\end{array}$} & \\
\hline & Pre-HTA & 105 & 3,70 & 338 & 18,50 & 443 & 9,40 & & \\
\hline & PA normal & 589 & 20,60 & 396 & 21,60 & 985 & 21,00 & & \\
\hline & PA óptima & 2093 & 73,00 & 853 & 46,60 & 2946 & 62,70 & & \\
\hline \multirow[t]{2}{*}{ Colesterol HDL } & Bajo & 1444 & 50,40 & 1582 & 86,40 & 3026 & 64,40 & \multirow{2}{*}{$\begin{array}{l}633,90 \\
p=0,00\end{array}$} & \multirow[t]{2}{*}{6,28} \\
\hline & Normal & 1422 & 49,60 & 248 & 13,60 & 1670 & 35,60 & & \\
\hline \multirow[t]{2}{*}{ Hipertrigliceridemia } & Sí & 510 & 17,80 & 1466 & 80,10 & 1976 & 42,10 & \multirow{2}{*}{$\begin{array}{l}1779,41 \\
p=0,00\end{array}$} & \multirow[t]{2}{*}{18,60} \\
\hline & No & 2356 & 82,20 & 364 & 19,90 & 2720 & 57,90 & & \\
\hline \multirow{3}{*}{ Glicemia } & Diabetes & 23 & 0,80 & 91 & 5,00 & 114 & 2,40 & \multirow{3}{*}{$\begin{array}{l}800,13 \\
p=0,00\end{array}$} & \\
\hline & Prediabetes & 107 & 3,70 & 572 & 31,30 & 679 & 14,50 & & \\
\hline & Normal & 2736 & 95,50 & 1167 & 63,80 & 3903 & 83,10 & & \\
\hline \multirow[t]{2}{*}{ Obesidad central } & Sí & 2194 & 76,60 & 1784 & 97,50 & 3978 & 84,70 & \multirow{2}{*}{$\begin{array}{l}377,88 \\
p=0,00\end{array}$} & \multirow[t]{2}{*}{11,88} \\
\hline & No & 672 & 23,40 & 46 & 2,50 & 718 & 15,30 & & \\
\hline
\end{tabular}

En cuanto al perfil epidemiológico que se observa en la tabla 3, vemos que el SM se presentó con mayor frecuencia en el sexo femenino $(68,10 \%)$ y en el grupo etario "adulto maduro" $(48,30 \%)$. 
Yeny Ofelia Tejada López; Gloria María Choquehuanca Zambrano; Evelyn del Socorro Goicochea Ríos; Jessica Ericka Vicuña Villacorta; Olga Yanet Guzmán Aybar

Tabla 3. Perfil epidemiológico del síndrome metabólico en adultos atendidos en el HIFM EsSALUD-Trujillo, 2014-2017

\begin{tabular}{|c|c|c|c|c|c|}
\hline \multirow[t]{2}{*}{ Variables } & & & \multicolumn{2}{|c|}{$\begin{array}{l}\text { Diagnóstico de } \\
\text { síndrome metabólico }\end{array}$} & \multirow[t]{2}{*}{ Total } \\
\hline & & & No & Sí & \\
\hline \multirow[t]{4}{*}{ Sexo } & Femenino & $\mathrm{N}^{\circ}$ & 2042 & 1246 & 3288 \\
\hline & & $\%$ & 71,20 & 68,10 & 70,00 \\
\hline & Masculino & $\mathrm{N}^{\circ}$ & 824 & 584 & 1408 \\
\hline & & $\%$ & 28,80 & 31,90 & 30,00 \\
\hline \multirow[t]{4}{*}{ Antecedente HTA } & No & $\mathrm{N}^{\circ}$ & 2182 & 1348 & 3530 \\
\hline & & $\%$ & 76,10 & 73,60 & 75,20 \\
\hline & Sí & $\mathrm{N}^{\circ}$ & 684 & 482 & 1166 \\
\hline & & $\%$ & 23,90 & 26,30 & 24,80 \\
\hline \multirow[t]{4}{*}{ Antecedente DM2 } & No & $\mathrm{N}^{\circ}$ & 2198 & 1261 & 3459 \\
\hline & & $\%$ & 76,70 & 68,90 & 73,60 \\
\hline & Sí & $\mathrm{N}^{\circ}$ & 668 & 569 & 1237 \\
\hline & & $\%$ & 23,30 & 31,10 & 26,30 \\
\hline \multirow[t]{8}{*}{ Edad } & Joven & $\mathrm{N}^{\circ}$ & 12 & 0 & 12 \\
\hline & & $\%$ & 0,40 & 0,00 & 0,30 \\
\hline & Adulto joven & $\mathrm{N}^{\circ}$ & 1423 & 553 & 1976 \\
\hline & & $\%$ & 49,70 & 30,20 & 42,10 \\
\hline & Adulto maduro & $\mathrm{N}^{\circ}$ & 1006 & 883 & 1889 \\
\hline & & $\%$ & 35,10 & 48,30 & 40,20 \\
\hline & Adulto mayor & $\mathrm{N}^{\circ}$ & 425 & 394 & 819 \\
\hline & & $\%$ & 14,80 & 21,50 & 17,40 \\
\hline
\end{tabular}

\section{DISCUSIÓN}

En los últimos años, los diversos cambios tecnológicos, económicos y sanitarios en nuestra sociedad han traído consigo el incremento del sedentarismo, la alimentación alta en carbohidratos y grasas saturadas $(9,21,22)$, el estrés laboral y la disminución del ejercicio físico ${ }^{(21,22)}$. Como consecuencia de ello, también se ha incrementado el número de diversas enfermedades metabólicas que inicialmente pasan desapercibidas para el paciente, e incluso son infradiagnosticadas, y pueden ser un verdadero peligro para su vida. En este contexto, el síndrome metabólico (SM) representa un conjunto de manifestaciones características, cuyo reconocimiento y reporte de prevalencia es de gran importancia para un abordaje integral en el centro sanitario correspondiente.

En el presente estudio encontramos SM en, aproximadamente, 39 de cada 100 pacientes, la mayoría de ellas mujeres mayores de 40 años. Según los criterios FID, esta prevalencia $(38,97 \%)$ concuerda con el reporte de Pacheco et al. (20) en México, quienes encontraron el $63 \%$ de prevalencia de síndrome metabólico en pacientes adultos; en España, Fernández et al. ${ }^{(14)}$, mostraron un valor de $58,2 \%$, y en Perú, Adams et al. ${ }^{(23)}$ reportaron una prevalencia de 40,1\%. Este hallazgo podría deberse al incremento constante en el consumo de bebidas azucaradas y de alimentos ricos en grasa, e ingesta de comida rápida que son habituales en estos países ${ }^{(9,21,22)}$.

En cuanto al perfil clínico conformado por los criterios diagnósticos del SM, el más frecuente fue la obesidad central con un $97,50 \%$, que también fue reportada como el principal criterio por Diaz $(72,2 \%)^{(24)}$ y por Aliaga et al. $(35,3 \%)^{(6)}$. Aunque en los trabajos de Castañeda ${ }^{(4)}$ y Palomino ${ }^{(7)}$ fue la tercera de los cinco criterios en frecuencia, la prevalencia fue similar con el $77,5 \%$ y $45,97 \%$ de individuos, respectivamente. Nuestra mayor prevalencia se atribuiría a que los criterios IDF 
empleados en nuestro estudio tienen un corte menor para obesidad con respecto a los criterios ATP III utilizados en los trabajos comparados ${ }^{(23)}$. Cabe recalcar lo importante que es su reconocimiento y tratamiento oportuno, pues se sabe que la obesidad central es la causante de las alteraciones de los demás criterios y es el principal factor de riesgo cardiovascular. Su mecanismo de daño se atribuye a que el tejido graso acumulado en el hígado, músculos y páncreas ${ }^{(2)}$ libera adipoquinas que favorecen los estados proinflamatorios y protrombóticos $(2,19)$, y producen lipotoxicidad e incrementan la producción de óxido nítrico, lo que altera la función de las células beta $y$, finalmente, produce su apoptosis (1), lo que bloquea a los receptores insulínicos y los GLUT-4 por fosfarilización, impide la captación de glucosa por el músculo ${ }^{\left({ }^{(1)}\right)}$ y ocasiona la hiperinsulinemia y la resistencia a la insulina ${ }^{(1,2,19)}$. Así mismo, diversos estudios han demostrado la efectividad del ejercicio físico y baja de peso en la disminución de la insulinoresistencia ${ }^{(25)}$.

El segundo criterio en frecuencia es el colesterol HDL por debajo del rango normal, con una prevalencia del $86,40 \%$, lo que coincide con lo reportado por Castañeda ${ }^{(4)}$ y Palomino ${ }^{(7)}$ quienes también lo hallaron en segundo lugar con $82,7 \%$ y $81,05 \%$ de prevalencia, respectivamente. El HDL tiene una función antioxidante y antinflamatoria ${ }^{(2)}$, e interviene en el transporte reverso del colesterol. Se ha descrito que niveles mayores a $40 \mathrm{mg} / \mathrm{dL}$ son un factor protector contra el proceso aterotrombótico ${ }^{(26)}$. Su producción es estimulada por el consumo de aceites vegetales y frutos secos en gran proporción, por lo que es esencial incluirlos en la dieta que acompaña al tratamiento.

La hipertrigliceridemia es un tipo de dislipidemia no aterogénica (cuando se presenta de manera aislada) y aterogénica (si está acompañada de otra dislipidemia) (23). Se presenta con el aumento de la producción hepática de lipoproteínas de muy baja densidad (VLDL, por sus siglas en inglés very low density lipoprotein ) y se relaciona con la obesidad y el SM, colabora en la disminución del colesterol de alta densidad HDL, la asociación de ambas es frecuente (27). En nuestra investigación, la hipertrigliceridemia es la tercera enfermedad en frecuencia, con una prevalencia del $80,10 \%$. Estudios similares revelan su prevalencia como la primera con un $91,8 \%{ }^{(4)}$, o segunda con un $48,39 \%$ y $28,3 \%$ por Palomino et al. ${ }^{(7)}$ y Avalos et al. ${ }^{(8)}$, respectivamente, lo que demuestra que es importante considerarla para elegir el tratamiento integrado.

Otro de los criterios hallado en los pacientes con SM es la presión arterial más elevada que los parámetros normales lo cual se relaciona con el incremento de peso (28), la edad (26), la hiperglicemia y la hiperinsulinemia; lo que hace que falle el sistema renina angiotensina ${ }^{(3)}$, el transporte de sodio en el riñón, que aparezca el daño endotelial y, en consecuencia, la arteroesclerosis ${ }^{(28)}$. En nuestra población encontramos una prevalencia de $26,3 \%$, lo que no concuerda con lo reportado por Castañeda (4) quien halló que, de 210 pacientes, el 74,59\% tenían antecedentes de HTA a pesar de haber tamizado a población muy parecida en edad y etnia. Es muy probable que esta diferencia se deba a que nuestra población fue más numerosa y con predominio de adultos mayores.

Dentro del perfil epidemiológico, el SM es más frecuente en el sexo femenino, lo cual se relaciona con encontrado por Delgado (26) y Aliaga et al. (6) ; y difiere de Gotthelf et al. (11) y Fernández et al. (14) que mencionan que el cuadro es más frecuente en los hombres. Esta diferencia se debe cual es el grupo predominante en cada población tamizada, en nuestro trabajo las mujeres son las que asisten al hospital con mayor frecuencia; además a esto se sumaría que la menopausia predispone al acumulo de tejido graso visceral y dislipidemias ${ }^{(3)}$.

Vemos que el SM presenta una elevada prevalencia en nuestra población, lo que nos obliga a mejorar los métodos de diagnóstico tanto clínicos como de laboratorio, y considerar métodos de tamizaje temprano en nuestras instituciones $y$, si bien no es considerado una enfermedad propiamente dicha, deben tenerse en mente medidas terapéuticas tempranas para reducir el riesgo de futuros eventos clínicos que pongan en riesgo la calidad de vida del paciente.

Para concluir, debemos señalar que, en nuestra investigación, más de un tercio de la población estudiada tiene SM, y que los criterios que predominaron fueron obesidad, colesterol HDL bajo, e hipertrigliceridemia. El SM es una condición fisiopatológica que se asocia al desarrollo de múltiples enfermedades metabólicas y cardiacas, y es más frecuente en el grupo de "adultos maduros" y en las mujeres. La investigación exhaustiva de los diversos perfiles clínicos en nuestro medio es fundamental para la futura capacitación e implementación de nuestros programas de atención integral en salud.

Contribución de los autores: Yeny Ofelia Tejada López y Gloria María Choquehuanca Zambrano estuvieron a cargo la recolección y tabulación y análisis de datos. Evelyn del Socorro Goicochea Ríos participó en la redacción, el análisis crítico y la aprobación final del artículo. Olga Guzmán Yabar se encargó de la asesoría de la investigación. Jessica Ericka Vicuña Villacorta contribuyó en la revisión de la base de datos y en el análisis estadístico.

Fuentes de financiamiento: Este artículo ha sido financiado por los autores.

Conflicto de interés: Los autores declaran no tener conflictos de interés. 


\section{REFERENCIAS BIBLIOGRÁFICAS}

1. Lahsen R. Síndrome metabólico y diabetes. Rev Med Clin Las Condes. 2014; 25(1): 47-52.

2. Lizarzaburu Robles JC. Síndrome metabólico: concepto y aplicación práctica. An Fac Med. 2013; 74(4): 315-20.

3. Sánchez Bustamante SJ. Prevalencia del síndrome metabólico y factores asociados, en usuarios del centro de salud Velasco Ibarra, octubre 2018 - abril 2019 [Tesis de pregrado]. Machala: Universidad Técnica de Machala. Facultad de Ciencias Químicas y de la Salud; 2019.

4. Castañeda Tirado WA. Perfil clínico-epidemiológico del síndrome metabólico en adultos del Hospital Víctor Lazarte Echegaray Trujillo - Perú 2013 [Tesis de pregrado]. Trujillo: Universidad Privada Antenor Orrego. Facultad de Medicina Humana; 2014.

5. Salinas Méndez LE, Vargas Álvarez JE, Mendoza Sánchez K, PuigNolasco A, Puig-Lagunes AA. Prevalencia y factores de riesgo del desarrollo de síndrome metabólico y prevalencia en estudiantes universitarios. Rev Cubana Invest Bioméd. 2018; 37(1).

6. Aliaga E, Tello T, Varela L, Seclén S, Ortiz P, Chávez H. Frecuencia de síndrome metabólico en adultos mayores del Distrito de San Martín de Porres de Lima, Perú según los criterios de ATP III y de la IDF. Rev Med Hered. 2014; 25: 142-8.

7. Palomino Baldeon JC, Navarro Chumbes GC. Síndrome metabólico y puesto de trabajo. Med Segur Trab. 2010; 56(221): 280-7.

8. Avalos Ibáñez KL, Caballero Rodríguez SA. Síndrome metabólico en adulto que acuden al laboratorio Quintanilla en Trujillo, febreromarzo 2017 [Tesis de pregrado]. Trujillo: Universidad Nacional de Trujillo. Facultad de Farmacia y Bioquímica; 2017.

9. Asociación Latinoamericana de Diabetes. Guías ALAD sobre el diagnóstico, control y tratamiento de la diabetes mellitus tipo 2 con medicina basada en evidencia. Edición 2019. Disponible en: http://revistaalad.com/guias/5600AX191_guias_alad_2019.pdf

10. Soto Pascual M, Bernui Leo I, Carbajal Gómez I. Prevalencia de síndrome metabólico en trabajadores del centro materno infantil Miguel Grau-Chaclacayo-Perú. An Fac Med. 2015; 76(2): 155-60.

11. Gotthelf S, Tempestti C, Rivas P. Síndrome metabólico y nivel educativo en adultos de la ciudad de Salta 2017. Rev Fed Arg Cardiol. 2018; 47(1): 32-7.

12. Hernández-Cuervo $P$, Flores-Chávez A, Ramírez-Villafaña $M$, Dávalos-de la Cruz P, Romero-Moreno JR, González-Ponce F, et al. Síndrome metabólico en mujeres derechohabientes del Instituto Mexicano del Seguro Social: prevalencia y factores asociados. Rev El Residente. 2016; 11(1): 3-9.

13. Gómez Torres FD, González Lemoine M, Legrá Sevila M, Pereña Haber L, López Herrera A. Prevalencia del síndrome metabólico en población de 15 a 74 años del municipio Guantánamo. Rev Inf Cient. 2017; 96(3): 454-65.

14. Fernández-Ruiz VE, Paniagu-Urbano JA, Solé-Agustí M, RuizSánchez A, Gómez-Marín J. Prevalencia de síndrome metabólico y riesgo cardiovascular en un área urbana de Murcia. Nutr Hosp. 2014; 30(5): 1077-83.

15. Castillo Hernández L, Cuevas González MJ, Almar Galiana M, Romero Hernández EY. Síndrome metabólico, un problema de salud pública con diferentes definiciones y criterios. Rev Méd Univ Veracruzana. 2017; 17(2).

16. Rosas Guzmán J, González Chávez A, Aschner P, Bastarrachea R. Epidemiología, diagnóstico, control, prevención y tratamiento de síndrome metabólico en adultos. Asociación Latinoamericana de Diabetes. pp. 25-44.

17. Carbajal Carvajal C. Síndrome metabólico: definiciones, epidemiología, etiología, componentes y tratamiento. Rev Med Leg Costa Rica. 2017; 34(1).

18. Fernández-Travieso JC. Síndrome metabólico y riesgo

cardiovascular. Rev CENIC Ciencias Biológicas. 2016; 47(2): 106-19.

19. Pereira-Rodríguez JE, Melo-Ascanio J, Caballero-Chavarro M, Rincón-Gonzales G, Jaimes-Martín T, Niño-Serrato R. Síndrome metabólico. Apuntes de Interés. Rev Cubana Cardiol Cir Cardiovasc. 2016; 22(2): 1561-2937.

20. Pacheco-Armenta MC, Jáquez-Torres JA. Prevalencia de síndrome metabólico en la consulta externa. Rev Sanid Milit Mex. 2017; 71: 264-75.

21. Cabrera-Rode E, Stusser B, Cálix W, Orlandi N, Rodríguez J, CubasDueñas I, et al. Concordancia diagnóstica entre siete definiciones de síndrome metabólico en adultos con sobrepeso y obesidad. Rev Perú Med Exp Salud Pública. 2017; 34(1): 19-27.

22. Ninatanta-Ortiz JA, Núñez-Zambrano LA, García-Flores SA, Romani Romaní F. Frecuencia de síndrome metabólico en residentes de una región andina del Perú. Rev Perú Med Exp Salud Pública. 2016; 33(4): 640-50.

23. Adams KJ, Chirinos JL. Prevalencia de factores de riesgo para síndrome metabólico y sus componentes en usuarios de comedores populares en un distrito de Lima, Perú. Rev Peru Med Exp Salud Pública. 2018; 35(1): 39-45.

24. Díaz Arpi D. Prevalencia del síndrome metabólico en choferes de las empresas de transporte urbano del distrito José Domingo Choquehuanca - Azángaro, 2018 [Tesis de pregrado]. Puno: Universidad Nacional del Altiplano. Facultad de Enfermería; 2018.

25. Pollak F. Resistencia a la insulina: verdades y controversias. Rev Med Clin Las Condes. 2016; 27(2): 171-8.

26. Delgado Rivera GC. Prevalencia de síndrome metabólico en vendedores del mercado Santa Clara de Quito período febrero 2019 [Tesis de pregrado]. Quito; Universidad Central del Ecuador. Facultad de Ciencias Químicas; 2019.

27. Carranza-Madrigal J. Triglicéridos y riesgo cardiovascular. Med Int Méx. 2017; 33(4): 511-4.

28. Sánchez Amuy EM. Prevalencia de síndrome metabólico y factores de riesgo asociados en habitantes de la población del Juncal Imbabura-Ecuador, 2019 [Tesis de pregrado]. Ibarra: Universidad Técnica del Norte. Facultad de Ciencias de la Salud; 2019

\section{Correspondencia:}

Yeny Ofelia Tejada López

Dirección: David Lozano Manzana I lote 24 - Urb Bosque.

Trujillo, Perú.

Teléfono: 958400033

Correo electrónico: lapoeta17@gmail.com

Recibido: 04 de mayo de 2020

Evaluado: 22 de junio de 2020

Aprobado: 27 de agosto de 2020

(c) La revista. Publicado por Universidad de San Martín de Porres, Perú. (c) Br Licencia de Creative Commons Artículo en acceso abierto bajo términos de Licencia Creative Commons Atribución 4.0 Internacional. (http://creativecommons.org/licenses/by/4.0/)

\section{ORCID iDs}

Yeny Ofelia Tejada López

Evelyn del Socorro Goicochea Ríos

Olga Guzmán Yabar

Jessica Ericka Vicuña Villacorta Gloria María Choquehuanca Zambrano $\odot$ https://orcid.org/0000-0002-9518-3780
(- https: / / orcid.org/0000-0001-7959-3571 (1) https: / / orcid.org/0000-0001-9994-9184 - https: / / orcid.org/0000-0002-6467-3613

(1) https: / / orcid.org/0000-0002-8121-3790 